\title{
Evaluation of the Potential QT Effect of Iptakalim Hydrochloride, an Antihypertensive Drug, in Single- and Multiple-Ascending Dose Studies Using Concentration-QTc Analysis
}

\author{
Nan Bai ${ }^{1}$, Jin Wang ${ }^{1}$, Haoyue Che ${ }^{1}$, Hai Wang ${ }^{2}$, Rui Wang ${ }^{3}$, and Yun Cai ${ }^{1}$ \\ ${ }^{1}$ Chinese PLA General Hospital \\ ${ }^{2}$ Thadweik Academy of Medicine \\ ${ }^{3}$ Affiliation not available
}

June 11, 2020

\begin{abstract}
Background:Cardiotoxicity has been one of the most common causes for withdrawing drugs from the market, and its important manifestation is the prolongation of QT interval. Iptakalim hydrochloride (ITKL) is a selective ATP-sensitive potassium (KATP) channel opener, it is crucial to assess the risk of cardiac repolarization of ITKL in clinical trials. Objective: This study was conducted to determine the effect of ITKL on corrected QT (QTc) interval. Method: A randomized, double-blind, placebo-controlled, single- and multi-dose regimen was carried out to investigate the QTc and ITKL concentration correlation. ITKL was administered at doses of $5 \mathrm{mg}, 10 \mathrm{mg}, 15 \mathrm{mg}$, and $20 \mathrm{mg}$ with single oral administration and $10 \mathrm{mg}, 20 \mathrm{mg}$ with multiple oral administration, along with placebo, in 83 healthy subjects. Electrocardiograms (ECGs) and blood samples were collected on a preset time schedule. Result: The highest dose $(20 \mathrm{mg})$ of ITKL did not cause QTc prolongation with a predicted $\Delta \Delta \mathrm{QTcF}$ (Baseline and placebo-corrected QTc interval) effect under geometric Cmax of 1.67 milliseconds, meeting the criteria for negative effect on QT. The Exposure Response (ER) analysis indicated the effect of the drug on $\Delta \Delta \mathrm{QTcF}$ was relatively small. Conclusion: The upper bounds of the $90 \%$ confidence interval (CI) of the model-predicted $\Delta \Delta \mathrm{QTcF}$ at Cmax in all dose groups were bellow 10ms, suggesting that ITKL did not prolong QT interval.
\end{abstract}

Evaluation of the Potential QT Effect of Iptakalim Hydrochloride, an Antihypertensive Drug, in Single- and Multiple-Ascending Dose Studies Using Concentration-QTc Analysis

Nan Bai a\#, Jin Wang a\#, Haoyue Che ${ }^{a}$, Hai Wang ${ }^{\text {b* }}$, Rui Wang ${ }^{a^{*}}$, Yun Cai ${ }^{\text {a* }}$

${ }^{a}$ Medicine Clinical Research Center, Chinese People's Liberation Army General Hospital, Beijing 100853, China

${ }^{b}$ Cardiovascular Drug Research Center, Thadweik Academy of Medicine, Beijing 100039, China

\#Contribute equally to this work.

Runing title: C-QT study of Iptakalim Hydrochloride

Corresponding authors:

Hai Wang, Cardiovascular Drug Research Center, Thadweik Academy of Medicine, 27 Tai Ping Road, Beijing 100853, China.

Tel: 86-10-66931557, Fax: 86-10-66931557.

E-mail:linchuang2218@126.com 
Rui Wang, Center of Medicine Clinical Research, the PLA General Hospital, 28 Fu Xing Road, Beijing 100853, China.

Tel: 86-10-66939409, Fax: 86-10-88214425.

E-mail:wangrui301@vip.sina.com

Yun Cai , Center of Medicine Clinical Research, the PLA General Hospital, 28 Fu Xing Road, Beijing 100853, China.

Tel: 86-10-6693-6617, Fax: 86-10-8821-4425.

E-mail: caicai_hh@126.com

\begin{abstract}
Background: Cardiotoxicity has been one of the most common causes for withdrawing drugs from the market, and its important manifestation is the prolongation of QT interval. Iptakalim hydrochloride (ITKL) is a selective ATP-sensitive potassium (KATP) channel opener, it is crucial to assess the risk of cardiac repolarization of ITKL in clinical trials. Objective:This study was conducted to determine the effect of ITKL on corrected QT (QTc) interval. Method: A randomized, double-blind, placebo-controlled, singleand multi-dose regimen was carried out to investigate the QTc and ITKL concentration correlation. ITKL was administered at doses of $5 \mathrm{mg}, 10 \mathrm{mg}, 15 \mathrm{mg}$, and $20 \mathrm{mg}$ with single oral administration and $10 \mathrm{mg}, 20 \mathrm{mg}$ with multiple oral administration, along with placebo, in 83 healthy subjects. Electrocardiograms (ECGs) and blood samples were collected on a preset time schedule. Result:The highest dose (20mg) of ITKL did not cause QTc prolongation with a predicted $\Delta \Delta \mathrm{QTcF}$ (Baseline and placebo-corrected QTc interval) effect under geometric $\mathrm{C}_{\max }$ of 1.67 milliseconds, meeting the criteria for negative effect on QT. The Exposure Response (ER) analysis indicated the effect of the drug on $\Delta \Delta \mathrm{QTcF}$ was relatively small.Conclusion: The upper bounds of the $90 \%$ confidence interval (CI) of the model-predicted $\Delta \Delta \mathrm{QTcF}$ at $\mathrm{C}_{\max }$ in all dose groups were bellow 10ms, suggesting that ITKL did not prolong QT interval.
\end{abstract}

Study registry identification number: The study was registered at Chinese Clinical Trial Registry with registration number ChiCTR1800014466.

Key words: Iptakalim, QT interval, Exposure response, $\mathrm{C}_{\max }$.

\title{
What is already known about this subject:
}

- Iptakalim is a selective KATP channel opener exerting the blood pressure-lowering effect.

- Cardiotoxicity has been one of the most common causes for withdrawing drugs from the market.

- ER analysis has been recommended for the estimation of QT interval prolongation risks of drugs in appropriate research.

\section{What this study adds:}

- Demonstration that the new chemical entity Iptakalim does not produce clinically significant prolongation of cardiac repolarization.

- Demonstration the safety of chemical entity Iptakalim in single dose and multiple dose.

Hypertension is the most common chronic disease worldwide. It is one of the most important risk factors of cardiovascular and cerebrovascular diseases, causing major complications of stroke, myocardial infarction, heart failure and chronic kidney disease. Iptakalim (2,3-dimethyl- $N$-(1-methylethyl)-2-butanamine), discovered by the Institute of Pharmacology and Toxicology of the Academy of Military Medical Sciences, is a selective KATP channel opener ${ }^{[1]}$, which has undergone extensive pharmacological and electrophysiological studies. Pre-clinical studies have shown that it can reduce the endothelin levels in hypertensive state, inhibit the vasoconstricting effect of endothelin, inhibiting the expression of endothelin and endothelin converting enzymes, thereby exerting the blood pressure-lowering effect ${ }^{[2]}$. 
Cardiotoxicity has been one of the most common causes for withdrawing drugs from the market. An undesirable property of some non-antiarrhythmic drugs is their ability to delay cardiac repolarization, an effect that can be measured by the prolonged QT interval on the electrocardiogram. A delay in cardiac repolarization creates an electrophysiological environment that favors the development of cardiac arrhythmia, most clearly torsade de pointes (Tdp). A feature of Tdp is the pronounced prolongation of QT interval in the supraventricular beat preceding the arrhythmia. Tdp can degenerate into ventricular fibrillation, leading to sudden death.

The international Conference on Harmonization (ICH) issued a guidance in May 2005 on the Clinical Evaluation of QT/QTc Interval Prolongation and Proarrhythmic Potential for Non-Antiarrhymic Drugs (the ICH E14 document ${ }^{[3]}$ ), aiming to provide recommendations concerning the design, conduct, analysis, and interpretation of clinical studies to assess the potential of a drug to delay cardiac repolarization. Considering the drawbacks of conducting thorough QT/QTc (TQT) study, Consortium for Innovation and Quality in Pharmaceutical Development and the Cardiac Safety Research Consortium (IQ-CSRC) recommended the design and conduct of clinical study in healthy subjects using ER analysis to replace the TQT studies ${ }^{[4]}$. Subsequent studies successfully detected the QT effect of 5 drugs which are known to cause mild QT prolongation, leading to the revision of the ICH E14 guideline and the accompanying "Questions \& Answers: The Clinical Evaluation of QT/QTc Interval Prolongation and Proarrhythmic Potential for Non-Antiarrhythmic Drugs". Based on the growing experience in the ER analysis of TQT studies and the results of the IQ-CSRC study, the ICH E14 document was subsequently revised in December 2015 to describe the approach of using ER analysis applied to data from small studies as an alternative of TQT study. There are several studies using ER analysis in the estimation of QT interval prolongation risks of drugs ${ }^{[5,6]}$.

Moreover, understandings of the safety and pharmacokinetic (PK) profiles of new drugs are needed for clinical development; thus, we conducted a phase I study to investigate the safety, PK profile, and the effect on QT/QTc interval of ITKL in Chinese population.

1.

\section{Methods}

Study and ethics

The objectives of the single-ascending dose (SAD) and multiple-ascending dose (MAD) studies were to investigate safety, tolerability and PK of ITKL following single and multiple dosing and to initially predict whether ITKL had an effect on QT interval prolongation in healthy Chinese subjects by establishing an ER model. Both studies were double-blinded, randomized, and placebo-controlled. Part 1 followed a single oral dose in 60 healthy adult subjects, and part 2 followed multiple oral doses in 23 healthy adult subjects.

The study protocols were approved by the respective institutional review board (IRB) or Independent Ethics Committee (IEC). The studies were conducted in accordance with the Declaration of Helsinki (1989) and local applicable laws and regulations. All subjects provided written informed consent prior to their participation in the studies.

Study design and treatment

Both studies were double-blind, randomized, and placebo-controlled. Standard inclusion criteria for studies in healthy subjects were used, including heart rate (HR) between 60 and 100 beats per minute (bpm), systolic blood pressure between 90 and $140 \mathrm{mmHg}$, diastolic blood pressure between 60 and $90 \mathrm{mmHg}$, blood potassium between 3.5 and $5.5 \mathrm{mmol} / \mathrm{L}$, and QTc interval $<430$ milliseconds for men and $<450$ milliseconds for women. In addition, in the SAD study, 60 healthy men and women (30/30), aged between 18 and 44 years and with a body mass index (BMI) between 19.2 and $25.2 \mathrm{~kg} / \mathrm{m}^{2}$, were enrolled. Subjects were randomized to single oral doses of 5,10,15 or $20 \mathrm{mg}$ ITKL or placebo (active/placebo, 12/2 in 5 and $10 \mathrm{mg}$ groups, 12/4 in 15 and $20 \mathrm{mg}$ groups) following fasting for at least 10 hours. The in-house period lasted for 4 days and 3 nights, starting on the day before dosing. The administration of higher doses waited until the administration 
of lower dose was finished and the safety and tolerability assessment can support subsequent higher doses. In the MAD study, 23 healthy men and women (12/11), aged between 21 and 44 years and with a BMI between 19.4 and $25.9 \mathrm{~kg} / \mathrm{m} 2$, were enrolled. Subjects were randomized to multiple oral doses of 10 or 20 mg ITKL, following fasting for at least 10 hours. The in-house period lasted for 12 days and 11 nights, starting on the day before dosing, and subjects were administered on day 1 and days 3-9. Subjects dosed with placebo were analyzed as a pooled group. Demographic data of the 83 subjects in the SAD and MAD studies are summarized in Table 1. In all cohorts of the SAD and MAD study, treatment was administered with $240 \mathrm{ml}$ water under fasted condition in the morning, and subjects were not allowed to drink during the 2 hours before administration and 2 hours after administration. The subjects had standard meals 4 hours after dosing.

PK data collection

In the SAD study, approximately $4 \mathrm{ml}$ blood samples were collected before dosing (within $30 \mathrm{~min}$ ), and at $0.5 \mathrm{~h}, 1 \mathrm{~h}, 1.5 \mathrm{~h}, 2 \mathrm{~h}, 3 \mathrm{~h}, 4 \mathrm{~h}, 6 \mathrm{~h}, 8 \mathrm{~h}, 12 \mathrm{~h}, 24 \mathrm{~h}, 36 \mathrm{~h}$ after dosing. Indwelling catheters were used and $2 \mathrm{ml}$ of $0.9 \%$ physiological saline was used to flush lines after sample collection. Samples were centrifuged for $5 \mathrm{~min}$ at approximately $4^{\circ} \mathrm{C}$ and $2000 \mathrm{~g}$ for 30 minutes after collection. The supernatant (plasma) was divided into two parts. $1 \mathrm{ml}$ of the plasma was used for drug monitoring, and the remaining was kept as backup. Plasma samples were immediately frozen at -20 or lower, and they were uniformly transferred to a refrigerator below -70 for storage until PK analysis after the completion of one period.

In the MAD study, approximately $4 \mathrm{ml}$ blood samples were collected before dosing (within $30 \mathrm{~min}$ ), and at $0.5 \mathrm{~h}, 1 \mathrm{~h}, 1.5 \mathrm{~h}, 2 \mathrm{~h}, 3 \mathrm{~h}, 4 \mathrm{~h}, 6 \mathrm{~h}, 8 \mathrm{~h}, 12 \mathrm{~h}, 24 \mathrm{~h}, 36 \mathrm{~h}$ after dosing on day 1 , and at predose (within $30 \mathrm{~min}$ ), $2.5 \mathrm{~h}$ postdose on day $5^{\sim} 8$, and at predose (within $30 \mathrm{~min}$ ), $0.5 \mathrm{~h}, 1 \mathrm{~h}, 1.5 \mathrm{~h}, 2 \mathrm{~h}, 3 \mathrm{~h}, 4 \mathrm{~h}, 6 \mathrm{~h}, 8 \mathrm{~h}, 12 \mathrm{~h}, 24 \mathrm{~h}, 36 \mathrm{~h}$ postdose on day 9. Samples were processed as the same as in the SAD study.

An established and validated high-performance liquid chromatography tandem mass spectrometry method was used to determine the ITKL concentrations in plasma samples. The lower limit of quantification (LLOQ) was $0.5 \mathrm{ng} / \mathrm{ml}$. Calibration standard curve was linear over the range of $0.5 \mathrm{ng} / \mathrm{ml}$ to $200 \mathrm{ng} / \mathrm{ml}$, using a $1 / \mathrm{x}^{2}$ weighted least-squares linear regression analysis ( $\left.\mathrm{r}[?] 0.98\right)$. Concentrations below the lower limit of quantification were labeled as below limit of quantification (BLOQ). The inter-day assay accuracy (expressed as percent relative error, \%RE) determined from quality control (QC) samples (at concentrations of $1 \mathrm{ng} / \mathrm{ml}$, $10 \mathrm{ng} / \mathrm{ml}$ and $80 \mathrm{ng} / \mathrm{ml}$ ) ranged from $1.75 \%$ to $7.03 \%$. Assay precision (expressed as the inter-day percent relative standard error, \%RSD) ranged from $4.67 \%$ to $10.65 \%$.

\section{ECG data collection}

Twelve-lead ECGs were extracted at the prespecified times and analyzed by timepoint and with ER modeling. If the scheduled time for an ECG coincided with a blood collection, the ECG was performed at least 5 minutes prior to the blood collection. The rhythm, ventricular rate, PR interval, QRS complex, QT interval, and QTcF (QT values after Fridericia's correction) interval were collected, and analyzed by instrument and hand. The results of QTc were subject to the manual measurement of two cardiographers.

In the SAD study, ECGs were obtained at -30 min, -20 min, -10 min before dosing, and at 0.5h, $1 \mathrm{~h}, 1.5 \mathrm{~h}$, $2 \mathrm{~h}, 3 \mathrm{~h}, 4 \mathrm{~h}, 6 \mathrm{~h}, 8 \mathrm{~h}, 12 \mathrm{~h}, 24 \mathrm{~h}, 36 \mathrm{~h}$ after dosing, three times at each timepoint.

In the MAD study, ECGs were obtained at $-30 \mathrm{~min},-20 \mathrm{~min},-10 \mathrm{~min}$ before dosing, and at $0.5 \mathrm{~h}, 1 \mathrm{~h}, 1.5 \mathrm{~h}$, $2 \mathrm{~h}, 3 \mathrm{~h}, 4 \mathrm{~h}, 6 \mathrm{~h}, 8 \mathrm{~h}, 12 \mathrm{~h}, 24 \mathrm{~h}, 36 \mathrm{~h}$ after dosing on day 1 , and at predose (within $10 \mathrm{~min}$ ), 2.5h postdose on day $5 \sim 8$, and at $-30 \mathrm{~min},-20 \mathrm{~min},-10 \mathrm{~min}$ before dosing, and at $0.5 \mathrm{~h}, 1 \mathrm{~h}, 1.5 \mathrm{~h}, 2 \mathrm{~h}, 3 \mathrm{~h}, 4 \mathrm{~h}, 6 \mathrm{~h}, 8 \mathrm{~h}, 12 \mathrm{~h}, 24 \mathrm{~h}$, $36 \mathrm{~h}$ after dosing on day 9 .

In order to reduce the dependence of QT on HR, we used Fridericia's correction for all analyses.

$$
Q T c F=Q T / R R 0.33,(R R=60 / H R)
$$

Exposure (concentration)-response (QT/QTc) Model 
This study used nonlinear mixed effect method to establish ITKL drug plasma concentration and $\Delta$ QTcF model. According to the recommendations of scientific white paper ${ }^{[7]}$, if the five hypothesis (see left column of table 3) are met, the preset linear model can be used for analysis.

$\mathrm{QTcF}$ and $\Delta \mathrm{QTcF}$ calculation method is as follows:

$$
Q T \mathrm{cF}=\frac{Q T \text { intervel }}{\mathrm{RR}^{0.33}}
$$

Formula (1)

$$
Q T c F_{\mathrm{ijk}}=\mathrm{QTcF}_{\mathrm{ijk}}-\mathrm{QTcF}_{i, j, k=0}
$$

Formula (2)

$\mathrm{QTcF}_{\mathrm{ijk}}$ is the measurement point $\mathrm{k}$ of the ith subject in group $\mathrm{j}, \overline{\mathrm{QTcF}_{i, j, k=0}}$ is the baseline QTcF value of the ith subject, that is, the mean value of QTcF for the three times before administration.

\section{Basic model selection}

This study used nonlinear mixed effect method to establish drug plasma concentration (independent variables) and $\Delta \mathrm{QTcF}$ model. $\Delta \mathrm{QTcF}$ represents the $\mathrm{QTcF}$ value minus the average $\mathrm{QTcF}$, which is used as a dependent variable. The preset model of relations among drug plasma concentration and $\Delta \mathrm{QTcF}$ was the first choice. When model hypothesis 3 (no delay hypothesis) or hypothesis 4 (linear relation hypothesis) is rejected, a custom model is used for analysis. The custom model is as follows:

$$
Q T c F_{\mathrm{ijk}}=\left(\theta_{0}+\eta_{0, i}\right)+\theta_{1} \mathrm{TR}_{j}+\left(\theta_{2}+\eta_{2, i}\right) C_{\mathrm{ijk}}+\theta_{3} \mathrm{TIM} E_{j}+\theta_{4}\left(\mathrm{QTcF}_{i, j, k=0}-\mathrm{QTc} F_{0}\right.
$$

Formula (3)

The $\Delta \mathrm{QTcF}$ ijk and $\mathrm{C}_{\mathrm{ijk}}$ are the measurements of $\Delta \mathrm{QTcF}$ and drug plasma concentration, respectively, at time-point $\mathrm{k}$ of the ith subject in the first $\mathrm{j}$ group. $\vartheta_{0}$ is the typical intercept value of the group without treatment (placebo effect); $\vartheta_{1}$ is the fixed effect of group TRT $\left(j=0\right.$, placebo; $\mathrm{J}=1$, ITKL); $\vartheta_{2}$ is the typical value of the slope group estimated by the model; $\vartheta_{3}$ is the fixed effect of correction time factor; $\vartheta_{4}$ is the fixed effect of correction baseline; $\mathrm{QTcF}_{\mathrm{i}, \mathrm{j}=0}$ is the baseline $\mathrm{QTcF} ; \overline{\mathrm{QTcF}_{0}}$ is the mean of $\mathrm{QTcF}$ at time 0 (baseline) for all subjects. $\eta_{0}$, i and $\eta_{2}$, i are a respectively random effects between individual $\vartheta_{1}$ and $\vartheta_{2}$.

\section{Model selection of random effects}

Random model is also known as a statistical model, which describes the inter-individual random effect (variation) and intra-individual random effect (variation). According to formula (3), the addition of interindividual random effect is adopted:

Formula (4)

$\mathrm{P}_{\mathrm{TV}}$ is the typical values of a parameter group, $\mathrm{P}_{\mathrm{i}}$ is the individual parameter value, and $\eta_{\mathrm{i}}$ is the random effect between individuals, in line with normal distribution with a mean of 0 and a variance of $\omega^{2}$.

The additive model is used for intra-individual random effect (residual):

$Y_{o b s, i j}=Y_{p r e d, i j}+\varepsilon_{\mathrm{ij}}$ Formula (5)

$Y_{\text {obs,ij }}$ and $Y_{\text {pred,ij }}$ are the observed and predicted values of the effect, respectively.

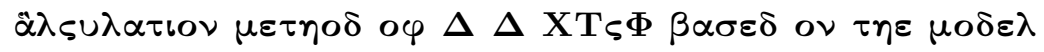

$\Delta \Delta \mathrm{QTcF}$, which is equal to the QTcF value minus the placebo and the average QTcF, is used for the baseline and the placebo effect correction. When the $\Delta \mathrm{QTcF}$ group typical values under the specific drug 
plasma concentrations is obtained by the model, the difference between $\Delta \mathrm{QTcF}$ group typical values and the $\Delta \mathrm{QTcF}$ group typical values in placebo group was calculated:

$$
\text { Mean } Q T c(C)=\operatorname{Mean}\left(Q T c_{\mathrm{ijk}} j=1, C_{\mathrm{ijk}}=C\right)-\operatorname{Mean}\left(Q T c_{\mathrm{ijk}} \mid j=0, C_{\mathrm{ijk}}=0\right)
$$

Formula (6)

$\mathrm{C}$ is the drug concentration (the geometric mean of each dose group $\mathrm{C}_{\max }$ ).

When the preset model is used, combining formula (3) and formula (6), the calculation formula of typical value of $\Delta \Delta$ QTCF group at a specific concentration, along with two-sided $90 \%$ CI can be obtained:

$$
\text { Estimated Mean } Q T c(C)=\theta_{1, E s t}+\theta_{2, E s t} * C
$$

Formula (7)

$$
\text { Estimated } S E=\sqrt{\operatorname{var}\left(\theta_{1, E s t}\right)+C^{2} * \operatorname{var}\left(\theta_{2, E s t}\right)+2 C *\left[\operatorname{cov}\left(\theta_{1, E s t} \theta_{2, E s t}\right)\right]}
$$

Formula (8)

$$
90 \% C I=\text { Estmated Mean } Q T c(C) \pm t \times \text { Estmated } S E
$$

Formula (9)

$\vartheta_{1 \text {,Est }}$ and $\vartheta_{2 \text {,Est }}$ are the intercept and slope estimated by the model, respectively; $\operatorname{var}\left(\vartheta_{1, \text { Est }}\right)$ and $\operatorname{var}\left(\vartheta_{2, \text { Est }}\right)$ are the inter-individual variances estimated by the model; $t$ is the boundary value at the corresponding significance level. In this study, the two-sided 90\% CI was calculated and the t value was 1.645.

When using a custom model, $\Delta \Delta$ QTcF group typical values under certain concentration are the value after the deduction of the baseline and time effect of the model. The two-sided $90 \%$ prediction interval (PI) calculation method used nonparametric Bootstrap resampling the parameter to calculate $\Delta \Delta \mathrm{QTcF}, 5^{\text {th }}$, $95^{\text {th }}$ points digits was used as the Bootstrap 90\% CI.

When the preset model were used, the $90 \%$ CI of $\Delta \Delta$ QTcF calculated by Bootstrap method were also used as auxiliary.

\section{Model evaluation method}

Goodness of Fit (GOF) was used to evaluate the skew or deviation (prediction errors) of the final model. Bootstrap method was used to verify the accuracy of the model. That is, 1000 new data sets are obtained by sampling the original data 1000 times, and the model parameters of each data set are calculated. The nonparametric statistical method is used to estimate the parameter points (median) and their $95 \%$ PI, that is, 2.5 and 97.5 quantiles of 1000 results.

1.

\section{Results}

Study population

All of the 60 (30 female and 30 male) and 23 (11 female and 12 male) healthy volunteers in each study group completed the treatment phases. The average age of each study group ranged from 28.8 to 33.5 years. Mean age and BMI were similar in male and female participants. A summary of the demographic characteristics of the subjects is presented in Table 1. 
Safety and PK analysis following ascending single and multiple oral doses

In the SAD study, the mean plasma concentrations of ITKL of the 48 healthy subjects after oral single dosage of $5 \mathrm{mg}, 10 \mathrm{mg}, 15 \mathrm{mg}$ and $20 \mathrm{mg}$ are presented in Figure 1a. The mean ITKL plasma concentrationtime profiles over the 36 -hour sampling time are presented. The PK profiles for all doses of ITKL under the fasted condition were characterized by a rapid absorption phase. The median $\mathrm{T}_{\max }$ ranged from $0.75 \mathrm{~h}$ to 1.50h. The median terminal elimination half-life $\left(\mathrm{t}_{1 / 2 \mathrm{z}}\right)$ was similar at each dose level, ranging from $6.66 \mathrm{~h}$ to 8.03h. Analysis of AEs in the study showed there were 13 cases (21.7\%) and 14 times of AE occurrence in all groups. There were 3 cases (25.0\%) 3 times in $5 \mathrm{mg}$ group, 1 case (8.3\%) 1 time in 10mg group, 4 cases $(33.3 \%) 5$ times in $15 \mathrm{mg}$ group, 3 cases $(25.0 \%) 4$ times in 20mg group, and 2 cases (16.7\%) 2 times in the placebo group. The incidence of AEs was not dose-dependent. All groups showed level I AEs.

In the MAD study, the mean plasma concentrations of ITKL of 23 healthy adults after oral multiple dosage of $10 \mathrm{mg}$ and $20 \mathrm{mg}$ are presented in Figure $1 \mathrm{~b}$. The median $\mathrm{T}_{\max }$ ranged from $1.25 \mathrm{~h}$ to $1.50 \mathrm{~h}$. The $\mathrm{t}_{1 / 2 \mathrm{z}}$ was similar at each dose level, ranging from $7.01 \mathrm{~h}$ to $7.91 \mathrm{~h}$. Among the AEs, 1 case in the $20 \mathrm{mg}$ group were lost to follow-up, and subject No. 607 in 10mg group was judged as level II AEs due to constipation and was treated with Enema Glycerin. Subject No. 711 was judged as level II AEs and treated with oral ammonium chloride Glycyrrhiza solution due to cough. The rest were level I AEs, but they were not related to the study drug. Except for one case which were lost to follow-up, the rest were all in remission and no SAEs and AEs leading to withdrawal from the study occurred.

$\Delta \Delta \mathrm{QTcF}$ and $\mathrm{C}_{\max }$ data of $\mathrm{SAD}$ and $\mathrm{MAD}$

Descriptive statistics $\mathrm{C}_{\max }$ and $\Delta \Delta \mathrm{QTcF}$ by timepoint are presented in Table 2 .

1. ER analysis

2. Model analysis

The drug plasma concentration data and QTc data of 83 patients with single and continuous administration were combined for model construction. A C-QTc model was established with the plasma concentration as the independent variable and $\Delta \mathrm{QTcF}$ as the dependent variable. When a research hypothesis is established, the preset linear model is preferred to describe the relationship between plasma concentration and $\Delta \mathrm{QTcF}$ (Table3): Hypothesis 1: since the $\Delta \Delta$ HR of subjects after administration is within $\pm 10 \mathrm{bpm}$ as shown in Figure 2a, it is judged that the drug has no effect on the HR of subjects; Hypothesis 2: there was no significant correlation between QTcF and HR, as shown in Figure 2b; Hypothesis 3: the $\mathrm{T}_{\max }$ of drug plasma concentration and $\Delta \mathrm{QTcF}$ in $10 \mathrm{mg}, 15 \mathrm{mg}$ and $20 \mathrm{mg}$ groups were all less than 1 hour, as shown in Figure $2 \mathrm{~d}$. The $\mathrm{T}_{\max }$ of $\Delta \mathrm{QTcF}$ in both $5 \mathrm{mg}$ and placebo groups was 6 hours, but the $\mathrm{T}_{\max }$ of drug plasma concentration of $5 \mathrm{mg}$ group was 1 hour. The facts that the drug plasma concentrations of the $5 \mathrm{mg}$ group did not change much between $1 \mathrm{~h}$ to $6 \mathrm{~h}$ and the $\mathrm{T}_{\max }$ of $\Delta \mathrm{QTcF}$ of the $5 \mathrm{mg}$ group and placebo group were the same suggested that the delay between plasma concentration of $5 \mathrm{mg}$ and $\Delta \mathrm{QTcF}$ may be caused by non-drug factors. Therefore, there is no delay in judging the change of blood concentration and $\Delta$ QTcF; Hypothesis 4: there is linear relationship between plasma concentration and $\Delta \mathrm{QTcF}$, as shown in Figure 2c; Hypothesis 5: the drug plasma concentrations of the single and multiple administration groups showed high consistence, because the same protocol and plasma analysis procedures were applied. Therefore, single and multiple administration studies can be combined for analysis.

Based on these data review, it is concluded that this study meets the five research hypotheses in Table 3, and can be analyzed using a preset linear model (Formula 3).

\section{Model evaluation}

The predicted mean $\Delta \Delta \mathrm{QTCF}$ at the observed geometric mean $\mathrm{C}_{\max }$ of the low dose of $5 \mathrm{mg}$ group and the high dose of $20 \mathrm{mg}$ group were calculated (Table 4). The two-sided 90\% CIs of the estimate were calculated using a bias-corrected nonparametric Bootstrap 1,000 resampling and subject is the unit of resampling. Table 5 lists the final model parameters. The success rate of fitting minimization was $97.6 \%$. The median 
and $95 \%$ PI of Bootstrap basically is coincide with the parameter estimation and 95\% CI, and the model shows high accuracy.

The GOF diagram of the final model is shown in Figure 2e. The regression trend line almost coincides with the standard line, and the conditional weight residual (CWRES) values are distributed between \pm 4 and uniformly distributed on both sides of the coordinate axis.

\section{Endpoint analysis}

$\Delta \mathrm{QTcF}$ variation over time is shown in Figure 3a. The specific changes of $\Delta \Delta \mathrm{QTcF}$ over time after merging single and multiple dosing data are shown in Figure 3b. It is concluded that ITKL would have no effect on QTc intervals, if the upper bound of the two-sided $90 \% \mathrm{CI}$ for predicted $\Delta \Delta \mathrm{QTcF}$ at the geometric mean $\mathrm{C}_{\max }$ of the high dose (20mg) group was no more than $10 \mathrm{~ms}$ according to preset modeling. The drug ITKL would have an effect on QTc intervals, if the upper bound of the two-side $90 \%$ CI for predicted $\Delta \Delta \mathrm{QTcF}$ at the geometric mean $\mathrm{C}_{\max }$ of the low dose $(5 \mathrm{mg})$ grou was more than $10 \mathrm{~ms}$ according to preset modeling.

The $\Delta \Delta \mathrm{QTCF}$ at the $\mathrm{C}_{\max }$ geometric mean of each dose group and its two-sided $90 \% \mathrm{CI}$ are calculated using the model formula. The results are shown in Table 2, Figure 3c and 3d. The upper limit of $90 \%$ CI of $\Delta \Delta \mathrm{QTcF}$ in each dose group was less than $10 \mathrm{~ms}$, indicating negative QT interval prolongation.

\section{Discussion}

Single dose PK and general safety profiles of ITKL have been studied in a previous phase I clinical trials ${ }^{[8]}$. Unlike previous studies, we included more healthy subjects (60) and added a 15mg dose group in this single dose study. Moreover, a multiple dose study with 23 subjects was also performed. ITKL showed linear PK characteristics in the dose range of $5 \mathrm{mg} \sim 20 \mathrm{mg}$ in Chinese healthy subjects. ITKL was eliminated from the body mainly through urine excretion. There was no significant difference between male and female in single dose exposures of $5 \mathrm{mg}$ and $10 \mathrm{mg}$, and there was a slight difference between men and women in single dose exposures of $15 \mathrm{mg}$ and 20mg. Multiple dosing study indicated continuous oral administration of ITKL tablets $10 \mathrm{mg}$ or $20 \mathrm{mg}$ basically had no accumulation effect. The incidence of AEs in both groups was high, but they were mild transient reactions, such as conjunctival congestion, decreased blood pressure, dizziness and so on, which may be related to the therapeutic mechanism of vasodilation by ITKL. There were no SAEs and AEs that led to withdrawal. Continuous oral administration of ITKL $10 \mathrm{mg}$ or $20 \mathrm{mg}$ was safe and well tolerated.

The effect of drugs on QT interval is often directly related to the drug plasma concentration.

ER analysis has enabled definitive evaluation of a drug's potential cardiotoxicity in standard clinical pharmacology studies, such as the first-in-human study, in which many small groups of subjects are given singleascending or multiple doses of the new chemical entity. Its application in the evaluation of cardiac safety of specific drugs is constantly affirmed ${ }^{[9]}$, and it is more efficient compared with conducting a stand-alone TQT study, as defined in the previous ICH E14 ${ }^{[10]}$. An assessment of the effects of Asenapine on QTc interval in patients with schizophrenia revealed a discrepancy between the results obtained by the intersection-union test: the intersection-union test can produce biased estimates resulting in a high false-positive rate in individual TQT studies. In such cases, simulation with an ER model can aid the reconciliation of the results from intersection-union test and this may be used to support the use of ER results as a basis for labeling ${ }^{[11]}$. On the other hand,recent studies have shown that the rate of false-negative results of ER model was acceptably low in placebo-controlled studies, especially for drugs with no or small QT effect, high plasma concentrations, and well-controlled variability of QT data ${ }^{[10]}$.

According to the white paper ${ }^{[7]}$ recommendations, this study meets all the applicability conditions of C-QT test exemption from traditional TQT research as described in the C-QT white paper which were showed as following: 1) According to the pre-clinical data of phase III, the clinical recommended dose of ITKL is $10 \mathrm{mg}$. The dose of $20 \mathrm{mg}$ in this trial group is twice of the clinical related exposure, which can ensure a low false-negative rate; 2) The QTc of ECG in this experiment was measured manually and interpreted by the 
technician of ECG room in a blind way. Before the interpretation, detailed SOP and corresponding training were carried out. The whole process was controlled by two person interpretation, and the interpretation result was reliable; 3) 83 healthy subjects were enrolled in the study, 60 cases of SAD were enrolled in the study, and they were divided into 4 dose groups (including 12 placebo) in the range of $5 \mathrm{mg} \sim 20 \mathrm{mg}$, each group was half male and half female; 23 cases of MAD were enrolled in the study, including 12 cases in the 10mg group (half male and half female), 11 cases in the 20mg group (6 male and 5 female), the sample size met the requirements; 4) For model analysis, the planned blood sample and ECG collection time shall prevail, When the difference between the actual time and the planned time is within $\pm 5 \mathrm{~min}$, the difference is within the range allowed by the time window, and the planned time is used to calculate. In this study, no data beyond the time window is found, which meets this condition; 5) After administration, $\Delta \Delta \mathrm{HR}$ was within $\pm 10 \mathrm{bpm}$, which met this condition. So traditional TQT research can be exempted to assess the impact of drugs on QT interval. Thus, this study was conducted to demonstrate whether ITKL can affect QT intervals using ER analysis to assess the relationship between drug concentration and QTc. The results showed that the highest dose $(20 \mathrm{mg})$ of ITKL did not cause QT prolongation with a predicted $\Delta \Delta \mathrm{QTcF}$ effect of 1.67 milliseconds under geometric $\mathrm{C}_{\max }$, meeting the criteria for negative QT assessment. Using the results from ER analysis, the drug effect on $\Delta \Delta \mathrm{QTCF}$ associated with this concentration was expected to be relatively small. But there are some issues we should consider before we make conclusion showed in Table 2: first, there was hysteresis between concentration and $\triangle \mathrm{QTcF}$ in group $5 \mathrm{mg}$; secondly, we are worried about the QC of ECG reading. About the first point, although the difference value of Tmax of plasma concentration and $\triangle \mathrm{QTcF}$ was $5 \mathrm{~h}$, As we discussed above, we thought it was caused by non-pharmaceutical factors considering both of Tmax of $\Delta \mathrm{QTcF}$ in group $5 \mathrm{mg}$ and placebo were $6 \mathrm{~h}$; about the second point, ECG was read by two electro cardiographers, all ECG of one subject should be read in a blind method by one electro cardiographer, and the other one re-checks. To evaluate the consistency, two electro caidiographers was required to read 50 pieces of ECG before reading ECG of subjects. In summary, as we discussed with sponsor, we reached a consensus that the issues as mentioned above would not affect the result of the trial, so our conclusion is relatively reliable, that is, there was no substantial effect on QTcF prolongation observed following ITKL treatment through the dose range of $5 \mathrm{mg}, 10 \mathrm{mg}, 15 \mathrm{mg}$ and $20 \mathrm{mg}$.

ITKL is a more potent activator of the SUR2B/Kir6.1 subtype of KATP channels, this selectivity is not seen in other K-channel openers such as diazoxide or pinacidil ${ }^{[2]}$. KATP channels are ubiquitously expressed on the plasma membrane of cells in multiple organs, including the heart, pancreas and brain ${ }^{[12]}$. The molecular structure of KATP channels is thought to be a heteromultimeric (tetrameric) assembly of these complexes: Kir6.2 with SUR1 (SUR1/Kir6.2, pancreatic type), Kir6.2 with SUR2A (SUR2A/ Kir6.2, cardiac type), and Kir6.1 with SUR2B (SUR2B/Kir6.1, Vascular smooth muscle type) [i.e., (SUR/Kir6.x) 4] ${ }^{[13]}$. Diverse classes of KATP channels exist in different tissues and cell types. For example, sarcolemmal KATP channels in conduction system, smooth muscle and endothelium have the same pore-forming subunit (Kir6.1) and regulatory subunit (SUR2B) ${ }^{[14]}$. Iptakalim is a KATP channel opener with a unique chemical structure that differs from other KATP openers. Among the 3 different subtypes of KATP channels, Iptakalim exhibits significant selectivity for SUR2B/Kir6.1 channels (being absent in the atrium and ventricles), mild effects on SUR2A/Kir6.2 channels (existing in the atrium and ventricle), and fails to open SUR1/Kir6.2 channels (existing in the atrium). Therefore, Iptakalim has relatively little effect on atrium and ventricle. in vitro experiments showed that Iptakalim could selectively regulate gene expression in important organs, heart, brain and liver of rats, and repeated administration of ITKL did not lead to pharmacological changes of the heart, and no pathological changes were found in myocardial tissue and its ultrastructure ${ }^{[15]}$. The clinical trial with hypertension patients also showed that ITKL had significant antihypertensive effect, but no significant effect on QT interval dispersion ${ }^{[16]}$, which is consistent with our findings.

However, in another experimental system, it was showed that Iptakalim had an effect on the isolated heart of spontaneously hypertensive rats: $1 \mu \mathrm{mol} / \mathrm{L}$ showed simple HR acceleration, $10 \mu \mathrm{mol} / \mathrm{L}$ showed positive frequency and negative inotropic effect, $100 \mu \mathrm{mol} / \mathrm{L}$ showed simple negative inotropic effect ${ }^{[17]}$, this experimental effect in vitro may be related to the wild direct effect on SUR2A/Kir6.2 in high concentration Iptakalim environment. The effect on the heart of patients with hypertension under the normal concentra- 
tion of Iptakalim administration remains to be researched. And orther preclinical experiments showed that ITKL exhibits selective effects on cardiac function and hemodynamics in rats, but this effect is not obvious under normal blood pressure ${ }^{[18]}$. It is not clear whether this selectivity would be reflected in the AEs of QT interval prolongation until now. Therefore, we can only conclude that the therapeutic dose of ITKL has little effect on the cardiac QT interval of healthy adults, and its cardiotoxicity in patients with hypertension needs to be further explored.

\section{Conclusion}

In healthy people, ITKL appears safe when administered orally via single dose and multiple dose, and continuous oral administration of ITKL tablets 10mg or 20mg exhibited no accumulation effect. We used ER analysis to assess potential QT prolongation by ITKL. The upper bounds of the $90 \%$ CI of the modelpredicted $\Delta \Delta \mathrm{QTcF}$ effect at $\mathrm{C}_{\max }$ in all dose groups were below $10 \mathrm{~ms}$, suggesting that ITKL did not prolong QT interval.

\section{Declarations}

Author contributions

Dr Nan Bai designed the cardiac safety portions and SAD, MAD sudies, and oversaw their statistical analysis and interpretation. Ms Jin Wang assisted with interpretation of the data and performed the statistical analysis of the cardiac safety data. Ms Haoyue Che assisted in the interpretation of data and writing articles. Dr Hai Wang, Dr Rui Wang, were responsible for the overall conduct and interpretation of the two trials. Dr Yun Cai were responsible for data proofreading and writing articles.

Data Availability Statement

Research data are not shared.

Financial Disclosures and Competing Interests

Dr Rui Wang is the principal investigator of this clinical trial. Dr Hai Wang owns the patent of ITKL. Other authors have no financial disclosures or conflicts of interest to report.

The authors have not received funding from any sources other than their employing institutions, identified with the author's affiliations. The authors declare no other relationships or activities that might appear to have influenced the submitted work

\section{References}

1 Wang H. Pharmacological characteristics of the novel antihypertensive drug, iptakalim hydrochloride, and its molecular mechanisms. Drug Development Research 2003; 58: 65-8.

2 Pan Z, Huang J, Cui W, Long C, Zhang Y, Wang H. Targeting hypertension with a new adenosine triphosphate-sensitive potassium channel opener iptakalim. Journal of cardiovascular pharmacology 2010; 56: $215-28$.

$3<$ E14-Clinical-Evaluation-of-QT-QTc-Interval-Prolongation-and-Proarrhythmic-Potential-for-NonAntiarrhythmic-Drugs.pdf $>$.

4 Darpo B, Sarapa N, Garnett C, Benson C, Dota C, Ferber G, et al . The IQ-CSRC prospective clinical Phase 1 study: "Can early QT assessment using exposure response analysis replace the thorough QT study?". Annals of noninvasive electrocardiology : the official journal of the International Society for Holter and Noninvasive Electrocardiology, Inc 2014; 19: 70-81.

5 Kinrade SA, Mason JW, Sanabria CR, Rayner CR, Bullock JM, Stanworth SH, et al . Evaluation of the Cardiac Safety of Long-Acting Endectocide Moxidectin in a Randomized Concentration-QT Study. Clinical and translational science 2018; 11: 582-9. 
6 Matsukura S, Nakamura Y, Hoshiai K, Hayashi T, Koga T, Goto A, et al . Effects of moxifloxacin on the proarrhythmic surrogate markers in healthy Filipino subjects: Exposure-response modeling using ECG data of thorough QT/QTc study. Journal of pharmacological sciences 2018; 136: 234-41.

7 Garnett C, Bonate PL, Dang Q, Ferber G, Huang D, Liu J, et al . Scientific white paper on concentrationQTc modeling. Journal of pharmacokinetics and pharmacodynamics 2018; 45: 383-97.

8 Cai Y, Chai D, Pei F, Fang Y, Wang R, Liang BB, et al. Single-dose pharmacokinetics and safety of iptakalim hydrochloride in Chinese healthy volunteers. The Journal of pharmacy and pharmacology 2012; 64: $337-43$.

9 Tsamandouras N, Duvvuri S, Riley S. Impact of Phase 1 study design on estimation of QT interval prolongation risk using exposure-response analysis. Journal of pharmacokinetics and pharmacodynamics 2019; 46: 605-16.

10 Ferber G, Sun Y, Darpo B, Garnett C, Liu J. Study Design Parameters Affecting Exposure Response Analysis of QT Data: Results From Simulation Studies. Journal of clinical pharmacology 2018; 58: 674-85.

11 Chapel S, Hutmacher MM, Bockbrader H, de Greef R, Lalonde RL. Comparison of QTc data analysis methods recommended by the ICH E14 guidance and exposure-response analysis: case study of a thorough QT study of asenapine. Clinical pharmacology and therapeutics 2011; 89: 75-80.

12 Szeto V, Chen NH, Sun HS, Feng ZP. The role of K(ATP) channels in cerebral ischemic stroke and diabetes. Acta pharmacologica Sinica 2018; 39: 683-94.

13 Yokoshiki H, Sunagawa M, Seki T, Sperelakis N. ATP-sensitive K+ channels in pancreatic, cardiac, and vascular smooth muscle cells. The American journal of physiology 1998; 274: C25-37.

14 Foster MN, Coetzee WA. KATP Channels in the Cardiovascular System. Physiological reviews 2016; 96: $177-252$.

15 Wang Y. Analysis of Iptakalim-induced gene expression changes in heart, brain and liver by cDNA microarray. Chinese Pharmacological Bulletin 2007; 3.

16 Xuan ZL. Effects of iptakalim on antihypertensive effect and QT interval dispersion in patients with hypertension. Chinese Journal of Gerontology 2013; 17.

17 Liu W, Xiao WB, Lu XQ, Wang H. Effects of iptakalim on blood pressure and cardiac function in rats Chinese Pharmacological Bulletin 2003: 227-9.

18 Long CL. Effects of iptakalim on blood pressure and cardiac function in rats Chin J Clin Pharm Therap $2003 ; 5$.

Figure 1a. Mean plasma concentration (Mean+SD) by time point and dose level in the SAD study.

Figure 1b. Mean plasma concentration (Mean+SD) by time point and dose level in the MAD study.

Figure 2a. The curves of $\Delta \Delta$ HR-time curve of all cohorts (Mean $\pm \mathrm{SE}$ ).

Figure $2 \mathrm{~b}$. The scatter diagram of correction between QTcF and RR.

Figure 2c. The scatter diagram of correction between concentration and $\Delta \mathrm{QTcF}$.

Figure 2d. The Curves of concentration/ $\Delta \mathrm{QTcF}$-time of all cohort (Mean $\pm \mathrm{SE}$ ).

Figure 2e. The final model GOF diagram. (The black solid line is the standard line, and the red dotted line is the regression trend line).

Figure 3a. $\Delta$ QTcF variation over time.

Figure 3b. $\Delta \Delta$ QTcF variation over time. 
Figure 3c. The trend of plasma concentration versus $\Delta \Delta \mathrm{QTcF}$. The blue line is the predicted value of the model, and the shaded part is $90 \%$ confidence interval.

Figure 3d. $\Delta \Delta \mathrm{QTcF}$ and its $90 \% \mathrm{CI}$ at different doses. The blue interval is the interval calculated by the parameter method, and the red interval is the interval calculated by the Bootstrap method.
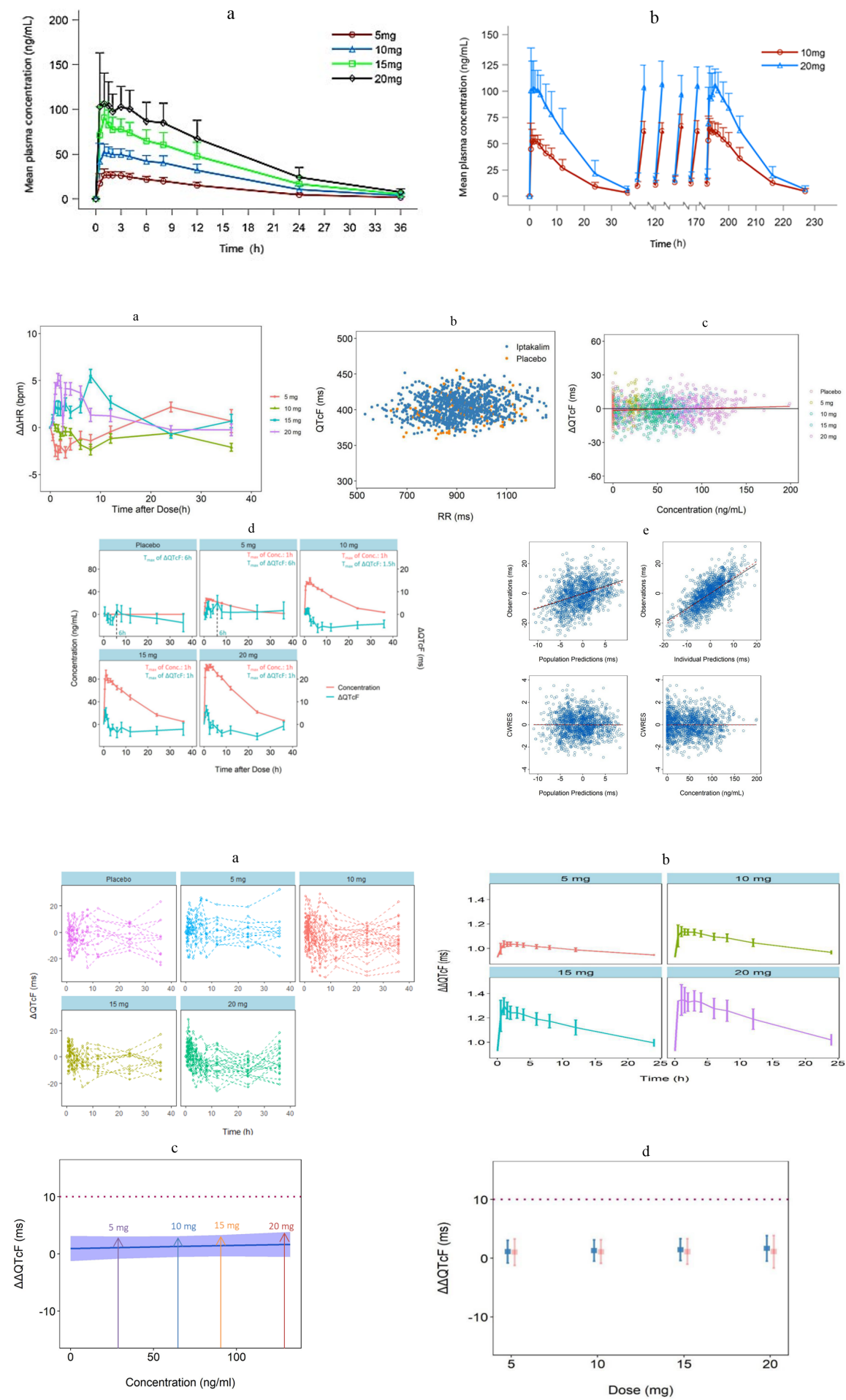


\section{Hosted file}

Table 1.docx available at https://authorea.com/users/332279/articles/458719-evaluation-ofthe-potential-qt-effect-of-iptakalim-hydrochloride-an-antihypertensive-drug-in-singleand-multiple-ascending-dose-studies-using-concentration-qtc-analysis

\section{Hosted file}

Table 2.docx available at https://authorea.com/users/332279/articles/458719-evaluation-ofthe-potential-qt-effect-of-iptakalim-hydrochloride-an-antihypertensive-drug-in-singleand-multiple-ascending-dose-studies-using-concentration-qtc-analysis

\section{Hosted file}

Table 3.docx available at https://authorea.com/users/332279/articles/458719-evaluation-ofthe-potential-qt-effect-of-iptakalim-hydrochloride-an-antihypertensive-drug-in-singleand-multiple-ascending-dose-studies-using-concentration-qtc-analysis

\section{Hosted file}

Table 4.docx available at https://authorea.com/users/332279/articles/458719-evaluation-ofthe-potential-qt-effect-of-iptakalim-hydrochloride-an-antihypertensive-drug-in-singleand-multiple-ascending-dose-studies-using-concentration-qtc-analysis

\section{Hosted file}

Table 5.docx available at https://authorea.com/users/332279/articles/458719-evaluation-ofthe-potential-qt-effect-of-iptakalim-hydrochloride-an-antihypertensive-drug-in-singleand-multiple-ascending-dose-studies-using-concentration-qtc-analysis 